\title{
Attenuating effect of casein glycomacropeptide on proliferation, differentiation, and lipid accumulation of in vitro Sprague-Dawley rat preadipocytes
}

\author{
S. P. Xu, X. Y. Mao, ${ }^{1}$ F. Z. Ren, and H. L. Che \\ Key Laboratory of Functional Dairy Science of Beijing and Ministry of Education, College of Food Science and Nutritional Engineering, \\ China Agricultural University, PO Box 303, Beijing 100083, China
}

\begin{abstract}
Food components with the ability to suppress preadipocyte proliferation and intracellular lipid accumulation may be helpful in the prevention of obesity, which is a worldwide health concern. Casein glycomacropeptide (GMP), which has pronounced gastric inhibitory activity, could potentially possess fat synthesis inhibition properties and an obesity-alleviating capacity. The objective of the present study was to investigate the effect of GMP on the proliferation and differentiation of preadipocytes as well as triglyceride accumulation and glycerol-3-phosphate dehydrogenase activity in preadipocytes isolated from Sprague-Dawley rats. Different dosages $(0,0.31,0.625,1.25,2.5$, and $5.0 \mathrm{mg} /$ $\mathrm{mL}$ ) of GMP were co-incubated with preadipocytes. The proliferation activity of preadipocytes significantly decreased in the GMP-treated group compared with that of the control group without GMP supplementation. The GMP exhibited an inhibitory effect against preadipocyte proliferation in a dose-dependent manner; the maximal antiproliferative effect was obtained with $2.5 \mathrm{mg} / \mathrm{mL}$. The GMP also attenuated differentiation, as revealed by decreased lipid content, and the effect was more pronounced when cells were treated with GMP before or at the beginning of differentiation induction than at later stages of cell differentiation. Cultured preadipocytes treated with GMP accumulated fewer triglycerides and had lower glycerol-3-phosphate dehydrogenase activity than did the control cells without GMP supplementation. In conclusion, GMP can inhibit the proliferation, differentiation, and lipid accumulation of preadipocytes in vitro.
\end{abstract}

Key words: casein glycomacropeptide (GMP), proliferation, differentiation, Sprague-Dawley rat preadipocyte

Received September 14, 2010.

Accepted October 13, 2010.

${ }^{1}$ Corresponding author: maoxueying@cau.edu.cn

\section{INTRODUCTION}

Obesity has become the leading metabolic disease in both developed and developing countries during recent years. A pathologic increase in the size and number of adipocytes and accumulation of fat are its main characteristics at the cellular level (Furuyashiki et al., 2004; Garaulet et al., 2006). The amount of adipose tissue can be regulated by the inhibition of adipogenesis and fat deposition (Rahman et al., 2008). It is well established that obesity can be attenuated not only by reduction of food intake due to the satiety-induction signal, but also by diminishing fat accumulation. Therefore, food components with the ability to suppress preadipocyte proliferation, differentiation, and intracellular lipid accumulation may be beneficial to body weight control and prevention of obesity.

Some researches have shown that flavonoids and phenolic acids (Hsu et al., 2006; Hsu and Yen, 2007), naringenin and hesperetin (Morikawa et al., 2008), and chitosan oligosaccharides (Rahman et al., 2008) inhibit the proliferation and adipogenesis of 3T3-L1 adipocytes. The ability of proteins and their hydrolysates to positively inhibit fat accumulation has been demonstrated in many in vitro experimental and animal models (Pilvi et al., 2007; Martinez-Villaluenga et al., 2009; Rajic et al., 2010). Soy protein hydrolysates enriched in $\beta$-conglycinin decreased lipid accumulation in 3T3-L1 adipocytes in vitro (Martinez-Villaluenga et al., 2009). An enriched bioactive fraction from whey protein isolate has been shown to inhibit adipocyte differentiation and lipid accumulation using both in vitro and in vivo models (Rajic et al., 2010). Whey-derived protein sources, particularly $\beta$-lactoglobulin-enriched fractions, have the ability to decrease both body weight gain and the adiposity index (Pichon et al., 2008). In addition, a high-calcium diet containing whey protein has shown to attenuate body weight and adipose tissue gain induced by a high-fat diet in an obese C57Bl/6J mice model (Pilvi et al., 2007). However, very little literature exists about the function of milk proteins and their hydrolysates on the proliferation and differentiation of preadipocytes. 
Dairy products and dairy components are known to suppress short-term food intake, increase subjective satiety, and stimulate the mechanisms known to signal satiation and satiety (Aziz and Anderson, 2007). Milk protein proteolysis products that are relevant to food intake regulation include casomorphins, casein glycomacropeptide (GMP), and the branched-chain amino acid leucine (Anderson and Moore, 2004). Casein glycomacropeptide is the C-terminal AA fragment of $\kappa$-casein released by chymosin by cleavage of the Phe105-Met106 peptide bond. Current research is investigating its proposed physiological activities, such as blood pressure lowering (Miguel et al., 2007), bacterial and viral adhesion inhibition (Brück et al., 2006), and bifidobacterial growth promotion (Azuma et al., 1984). The effect of GMP on the secretion of cholecystokinin (CCK) and induction of satiety has been reported (Burton-Freeman, 2008; Lam et al., 2009). For women, oral GMP may stimulate the release of CCK, a satiety-inducing peptide, and slow down gastric emptying (Yvon et al., 1994; Burton-Freeman, 2008). There is also an indication that whey is more satiating than is casein (Hall et al., 2003). Casein glycomacropeptide could inhibit gastric acid secretion in calves (Guilloteau et al., 1994). It also has been shown to be associated with reduced fat mass in Wistar rats (Royle et al., 2008). In addition, consumption of recombinant human GMP decreased the body weight gain, as well as liver, kidney, and adipose tissue weight of rats fed a high-fat diet (Kim et al., 2005). However, a study showed that GMP did not affect postprandial CCK concentrations, and the extent of GMP glycosylation had no influence on the results (Keogh et al., 2010). This controversy still needs to be settled.

Although GMP has been shown to stimulate the release of CCK, which may promote satiety and, thereby, decrease body weight and body fat mass, no data have shown whether GMP can regulate proliferation, differentiation, and adipogenesis of preadipocytes and adipocytes until now. We hypothesized that GMP could possess the particular property of fat synthesis inhibition and, thereby, decrease body weight. To further understand the contribution of GMP to body weight control, in the present study, we examined the inhibitory properties of GMP on the proliferation and differentiation, as well as lipid accumulation and glycerol-3-phosphate dehydrogenase (GPDH) activity of rat preadipocytes.

\section{MATERIALS AND METHODS}

\section{Reagents and Materials}

Reagents for preadipocyte culture, including Dulbecco's Modified Eagle Medium (DMEM), fetal bovine serum (FBS), HEPES, Ham's F-12 medium, penicillin, and streptomycin, were obtained from Gibco BRL Life Technologies Inc. (Grand Island, NY). Dexamethasone, 1-methyl-3-isobutylxanthine (MIX), insulin, L-glutamine, glucose, sodium pyruvate, Oil Red O, formalin, and 3-(4,5)-dimethylthiahiazo(-z-y1)-3,5-diphenytetrazoliumromide (MTT) were purchased from Sigma-Aldrich Co. (St. Louis, MO). Triglyceride (TG) and GPDH assay kits were acquired from Nanjing Jiancheng Bioengineering Institute (Nanjing, China). Casein glycomacropeptide with a purity of over $90 \%$ was separated from cheese whey as described previously (Ma et al., 2008).

\section{Cell Line and Cell Culture}

Primary rat preadipocytes were isolated from epididymal fat pads of male Sprague-Dawley rats as described previously (Forest et al., 1983). After collagenase digestion, the cell suspension was filtered through a $150-\mu \mathrm{m}$ nylon mesh filter to remove undigested tissue. The pellet obtained after centrifugation was resuspended in DMEM supplemented with $10 \%$ of FBS. The obtained rat preadipocytes can differentiate into adipocytes and increase lipid droplets in the cytoplasm following adipogenic stimulation as described previously (Torii et al., 2003). Preadipocytes were subcultured in DMEM with $10 \%$ of fetal bovine serum at $37^{\circ} \mathrm{C}$ in a $5 \% \mathrm{CO}_{2}$ atmosphere until $2 \mathrm{~d}$ postconfluence.

\section{Determination of Cell Proliferation and Viability by MTT Assay}

For cell proliferation, DMEM and Ham's F-12 medium ( $\mathrm{pH} 7.2$; containing $25 \mathrm{mmol} / \mathrm{L}$ HEPES) were used in a $1: 1$ ratio as the culture medium, and $4 \mathrm{mmol}$ of L-glutamine/L, $1.5 \mathrm{~g}$ of sodium bicarbonate/L, $4.5 \mathrm{~g}$ of glucose $/ \mathrm{L}, 1.0 \mathrm{mmol}$ of sodium pyruvate/L, $100 \mathrm{U}$ of penicillin $/ \mathrm{mL}, 100 \mu \mathrm{g}$ of streptomycin $/ \mathrm{mL}$, and $8 \%$ of FBS were added to the cell culture. To assess whether GMP could inhibit the proliferation of rat preadipocytes in a dose-responsive manner, preadipocytes were treated with GMP at concentrations of 0 (control group), $0.31,0.625,1.25,2.5$, or $5.0 \mathrm{mg} / \mathrm{mL}$. In order to evaluate the effect of treatment duration, preadipocytes were co-incubated with GMP at a concentration of 2.5 $\mathrm{mg} / \mathrm{mL}$ for $0,24,48,72$, and $96 \mathrm{~h}$. Cell growth was determined by an MTT assay according to the method as described previously (Mosmann, 1983). Briefly, cells were continuously cultured in the same medium with $0.1 \mathrm{mg}$ of $\mathrm{MTT} / \mathrm{mL}$ for $4 \mathrm{~h}$. After the culture medium was removed, cells were incubated in $0.1 \mathrm{~mL}$ of lysis solution. The MTT activity in each well was determined at a wavelength of $570 \mathrm{~nm}$. Antiproliferative activity was calculated using the following equation: 


$$
\begin{gathered}
\text { Antiproliferative activity (\%) } \\
=\left(1-\frac{\text { OD value of treated sample }}{\text { OD value of control }}\right) \times 100 \%,
\end{gathered}
$$

where OD is the optical density.

\section{Differentiation of Rat Preadipocytes}

Cell differentiation was induced using DMEM with $10 \%$ of fetal bovine serum supplemented with MDI solution (0.5 mM 1-methyl-3-isobutylxanthine, $1 \mu M$ dexamethasone, and $10 \mu \mathrm{g}$ of insulin $/ \mathrm{mL}$ ) as described previously (Guo et al., 2000). The GMP was added to cells at a concentration of $2.5 \mathrm{mg} / \mathrm{mL}$ to analyze the effect of GMP addition time on the differentiation of rat preadipocytes. Lipid droplets were visible by phasecontrast microscopy $2 \mathrm{~d}$ after MDI treatment.

\section{Lipid Quantification in Differentiated Preadipocytes by Oil Red O Assay}

In order to observe the effect of GMP on adipocyte differentiation and lipid accumulation, cells were further treated with GMP at a concentration of $2.5 \mathrm{mg} /$ $\mathrm{mL}$. Cultures were washed twice with PBS after $8 \mathrm{~d}$ of differentiation and fixed with $2 \mathrm{~mL}$ of $10 \%$ formalin (in PBS) for $1 \mathrm{~h}$. Cells were then washed twice with double distilled water and stained with $0.5 \%$ of Oil Red O for $15 \mathrm{~min}$ at room temperature (Wu et al., 1996). Absorbance at $510 \mathrm{~nm}$ was measured using a spectrophotometer. Inhibition of lipid accumulation in adipocytes was calculated using the following equation:

$$
\begin{aligned}
& \text { Lipid accumulation inhibitory activity (\%) } \\
& =\frac{\left(\text { absorbance }_{\text {control }}-\text { absorbance }_{\text {sample }}\right)}{\text { absorbance }_{\text {control }}} \times 100 \text {. }
\end{aligned}
$$

\section{Quantification of GPDH Activity and TG Content}

At the beginning of the differentiation period, GMP was added to preadipocytes at concentrations of 0 , $0.31,0.625,1.25,2.5$, and $5.0 \mathrm{mg} / \mathrm{mL}$, respectively. After $8 \mathrm{~d}$ of differentiation, the cells were harvested and washed twice with ice-cold PBS. The GPDH activity was determined according to the procedure as described previously (Wise and Green, 1979). Protein concentration was measured using the Lowry method (Lowry et al., 1951). One unit of enzyme activity was defined as the amount of protein required for oxidation of $1 \mathrm{nmol}$ of reduced nicotinamide adenine dinucleotide per minute. Triglyceride content was measured using a commercial available kit according to the manufacturer's instructions. The GPDH-attenuating activity and TG-decreasing activity were calculated according to the following equations:

$$
=\frac{\begin{array}{c}
\text { GDPH-attenuating activity }(\%) \\
\left(\text { absorbance }_{\text {control }}-\text { absorbance }_{\text {sample }}\right)
\end{array}}{\text { absorbance }_{\text {control }}} \times 100
$$

and

$$
=\frac{\left(\text { absorbance }_{\text {control }}-\text { absorbance }_{\text {sample }}\right)}{\text { absorbance }_{\text {control }}} \times 100 .
$$

\section{Statistical Analysis}

Results are expressed as mean \pm standard deviation of 3 replicated cultures. Data were analyzed using oneway ANOVA, followed by Duncan's multiple comparison test. All analyses were performed using SPSS version 13.0 software (SPSS Inc., Chicago, IL). The statistical significance level was set at $P<0.05$.

\section{RESULTS}

\section{Effect of GMP Concentration on Preadipocyte Proliferation}

The ability of GMP to inhibit preadipocyte proliferation is shown in Figure 1. Preadipocytes receiving GMP showed higher proliferation-decrease responses in comparison to those of the control cells (i.e., no GMP addition; $P<0.05$ ). The proliferation activity of preadipocytes treated with GMP was significantly decreased at all examined GMP concentrations in a dose-dependent manner up to $2.5 \mathrm{mg} / \mathrm{mL}$. The maximal antiproliferative concentration was $2.5 \mathrm{mg} / \mathrm{mL}$, which achieved $22.02 \pm 1.35 \%$ inhibition when compared with that of the control $(0 \mathrm{mg}$ of $\mathrm{GMP} / \mathrm{mL})$. When GMP concentration was greater than $2.5 \mathrm{mg} / \mathrm{mL}$, the antiproliferative activity did not increase significantly $(P$ $>0.05)$.

\section{Effect of Incubation Duration on the Antiproliferative Activity of GMP}

Casein glycomacropeptide was co-incubated with rat preadipocytes for $0,24,48,72$, and $96 \mathrm{~h}$. The antiproliferative activity of GMP increased when the length of incubation with preadipocytes was increased up to 72 


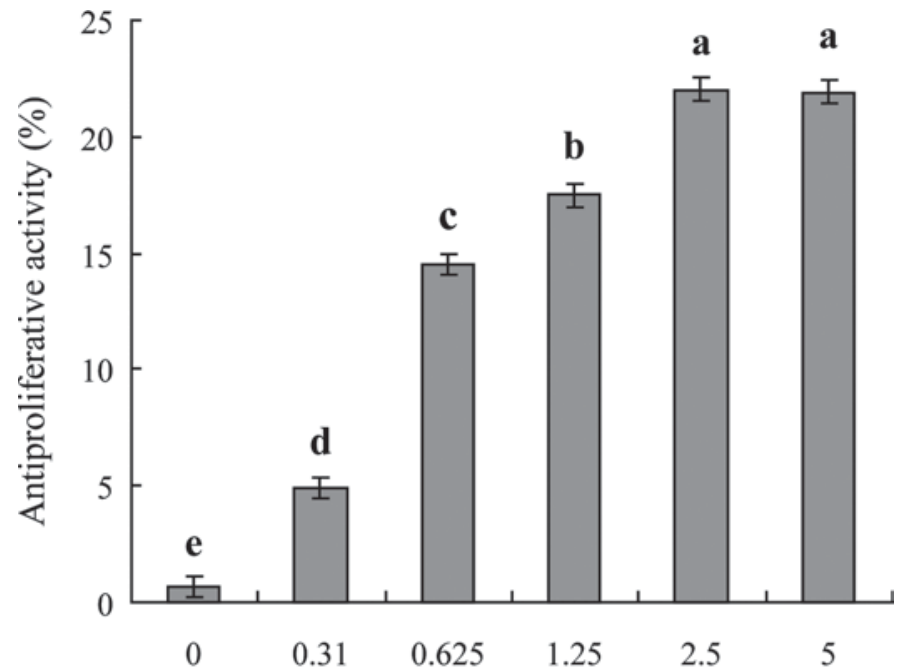

Concentration of GMP $(\mathrm{mg} / \mathrm{mL})$

Figure 1. Effect of various concentrations of casein glycomacropeptide (GMP) supplementation on rat preadipocyte proliferation, expressed as antiproliferative activity (\%). The values are presented as means $\pm \mathrm{SD}$ of 3 replicated cultures. Columns that do not share the same letter are significantly different from each other at $P<0.05$. Preadipocytes were treated with GMP at concentrations of 0 (control group), 0.31, 0.625, $1.25,2.5$, or $5.0 \mathrm{mg} / \mathrm{mL}$. The 3-(4,5)-dimethylthiahiazo(-z-y1)-3,5-di-phenytetrazoliumromide (MTT) activity was determined at a wavelength of $570 \mathrm{~nm}$.

$\mathrm{h}$ (Figure 2). The antiproliferative activity was greatest when co-incubation time was $72 \mathrm{~h}$. The GMP co-incubation time $(72 \mathrm{~h})$ was used for further antiproliferative activity studies.

\section{Effect of GMP Addition Time on Preadipocyte Proliferation}

The proliferation curve of preadipocytes without GMP is shown in Figure 3A. The growth of preadipocytes during the first $3 \mathrm{~d}$ was slow, which was termed the lag phase. After $3 \mathrm{~d}$ and until $6 \mathrm{~d}$, the growth of preadipocytes became rapid. This was termed the logarithmic growth phase. After $6 \mathrm{~d}$, the growth of preadipocytes became very slow, and was termed the stable phase. At different growth phases (i.e., lag, logarithmic, and stable phase), GMP was added to preadipocytes and co-incubated for $72 \mathrm{~h}$. The antiproliferative activity of GMP added at each of these phases is shown in Figure 3B. Adding GMP at the lag phase had the highest antiproliferative activity $(22.77 \pm 1.27 \%)$ compared with that of the logarithmic and stable phases (12.04 $\pm 0.35 \%$ and $6.34 \pm 0.22 \%$, respectively; $P<0.05$ ). This demonstrated that the antiproliferative activity of GMP depended on the phase of its addition, and treatment of preadipocytes with GMP at the early phase of cell proliferation (lag phase) markedly decreased preadipocyte proliferation.

\section{Effect of GMP Addition Time on Adipocyte Lipid Accumulation}

The natural differentiation curve of adipocytes without GMP addition is shown in Figure 4A. The defined stages are stage 1 (from $2 \mathrm{~d}$ before differentiation induction; i.e., $\mathrm{d}-2$ to $\mathrm{d} 0$ ), stage 2 (from d 0 to 2 ), stage 3 (from d 2 to 6 ), stage 4 (from d 6 to 10), and stage 5 (from d 10 to 14). The effect of GMP treatment during these stages on the lipid content of adipocytes is shown in Figure 4B. A decrease in lipid content was found for all GMP treatments, which was $36.38 \pm 1.27 \%, 38.92$ $\pm 0.35 \%, 30.53 \pm 0.42 \%, 28.27 \pm 0.36 \%$, and $15.33 \pm$ $0.34 \%$ when GMP was added at stages 1 to 5 , respectively, which suggests that adding GMP before or at the beginning (stage 1 and 2) of cell differentiation caused greater inhibition of lipid accumulation compared with that caused by GMP addition at later stages.

\section{Effect of GMP Concentration on Intracellular TG Content and GPDH Activity}

The effect of GMP concentration on differentiated adipocyte intracellular TG accumulation was determined, as shown in Figure 5A. Casein glycomacropeptide reduced intracellular TG accumulation in a dosedependent manner between 0 and $5.0 \mathrm{mg} / \mathrm{mL}$. Maximal TG reduction $(33.28 \pm 2.36 \%)$ was achieved with 2.5

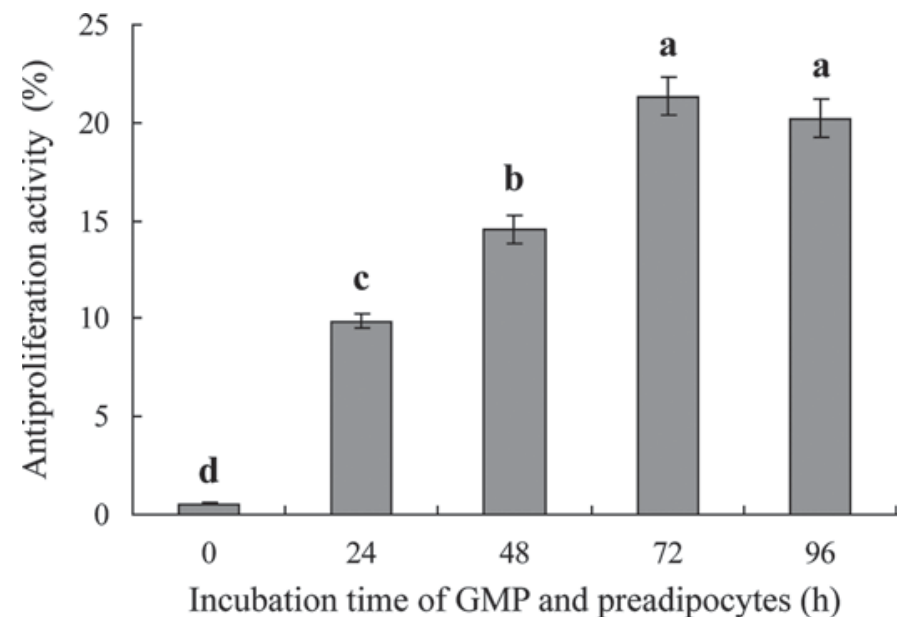

Figure 2. Decrease of rat preadipocyte proliferation (antiproliferative activity, \%) in response to casein glycomacropeptide (GMP) treatment at incubation time between 0 and $96 \mathrm{~h}$. The 3-(4,5)-dimethylthiahiazo(z-y1)-3,5-di-phenytetrazoliumromide (MTT) activity was determined at a wavelength of $570 \mathrm{~nm}$. The values are presented as means $\pm \mathrm{SD}$ of 3 replicated cultures. Columns that do not share the same letter are significantly different from each other at $P<0.05$. 
mg of GMP/mL supplementation. The addition of GMP also resulted in a marked decrease in GPDH activity in a similar dose-dependent manner (Figure 5B), which was $35.35 \pm 1.27 \%$ when adding GMP at a concentration of $1.25 \mathrm{mg} / \mathrm{mL}$.

\section{DISCUSSION}

The present work indicates that proliferation of preadipocytes significantly decreases with GMP treatment in a dose-dependent manner. The exposure of preadipocyte to GMP also significantly attenuated adipocyte differentiation in vitro, as revealed by decreased adipocyte lipid content, TG content, and GPDH activity, which indicated the inhibition of adipogenesis and adipocyte differentiation by GMP supplementation. Our results indirectly suggest that GMP supplementation may decrease the number and size of adipocytes. Casein glycomacropeptide has been shown to be associated with reduced fat mass in Wistar rats and has significant additional influence on fat accumulation when combined with whey protein isolate (WPI; Kim et al., 2005). Wang and Jones (2004) proposed that decreased preadipocyte proliferation and adipocyte lipogenesis are mechanisms of antiobesity. Whether GMP can attenuate fat accumulation induced by a high-fat diet of obese rats in vivo is still under further study in our laboratory.

This study shows that GMP addition at $2.5 \mathrm{mg} / \mathrm{mL}$ possesses the highest inhibition activity of preadipocyte proliferation and differentiation, as well as attenuating lipid accumulation. Chitosan oligosaccharides at the concentration of $4 \mathrm{mg} / \mathrm{mL}$ could significantly inhibit the differentiation of 3T3-1 adipocytes (Rahman et al., 2008). A high-protein diet $(300 \mathrm{~g} / \mathrm{kg})$ of whey protein concentrate was more effective than was red meat in reducing body weight gain and body fat content of rats when energy intakes were comparable (Belobrajdic et al., 2004). Carcass fat mass and body weight gain of Wistar rats fed WPI plus $200 \mathrm{~g}$ of GMP/ $\mathrm{kg}$ were significantly lower than that of rats fed WPI alone or casein-fed animals (Kim et al., 2005). Supplementation of recombinant human GMP lowered the body weight gain of male Sprague-Dawley rats fed a high-fat diet by 8.4 and $19.1 \%$ at doses of 0.5 and $1 \%$, respectively (Keogh et al., 2010). Therefore, the effective dosage of GMP remains to be clarified by rat models or human clinical trials.

Adipocytes are the primary sites for lipid accumulation and storage. Fat deposition in adipose tissue can be lowered by reducing lipid uptake in adipocytes via suppressing lipoprotein lipase or reducing lipid synthesis through inhibiting fatty acid synthase (FAS) (Li et al., 2008). Fatty acid synthase is an important enzyme in
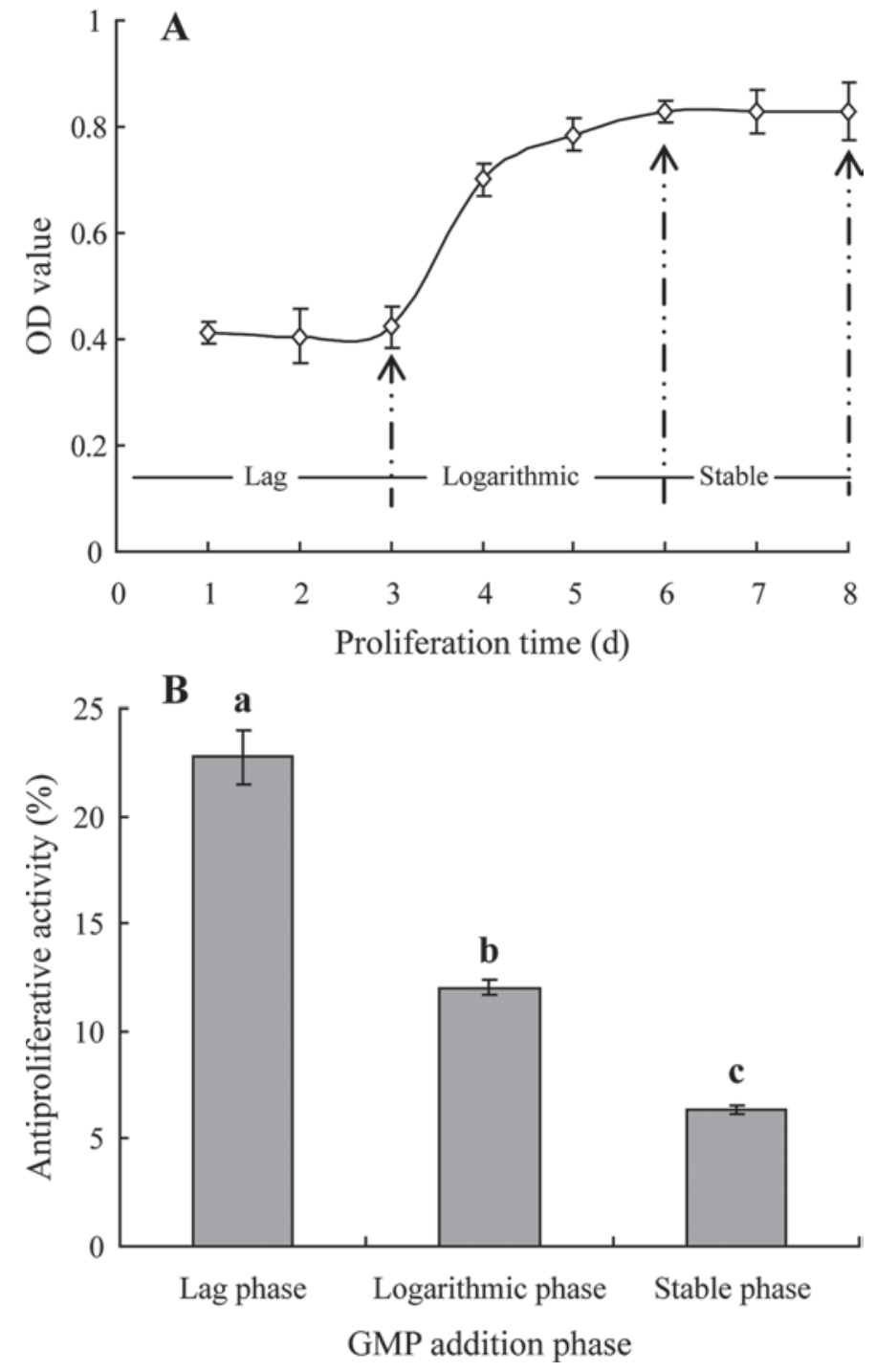

Figure 3. The proliferation curve of preadipocytes without casein glycomacropeptide (GMP) supplementation (A; OD = optical density). Casein glycomacropeptide $(2.5 \mathrm{mg} / \mathrm{mL})$ was added to preadipocyte culture at different phases of cell growth, and the decrease in rat preadipocyte proliferation was measured after incubation for $72 \mathrm{~h}(\mathrm{~B})$. The lag phase was from d 0 to 3 , the logarithmic phase from $\mathrm{d} 3$ to 6 , and the stable phase from d 6 to 8 . The values are presented as means $\pm \mathrm{SD}$ of 3 replicated cultures. Columns that do not share the same letter are significantly different from each other at $P<0.05$.

the pathway of fatty acid synthesis. Fatty acid synthase inhibitors have been reported to provide a potential pathway to target obesity therapy (Kuhajda et al., 2005). The present work shows that GMP can inhibit the differentiation of rat preadipocytes as well as attenuate lipid accumulation, which may indirectly mean that GMP can act as a FAS inhibitor. Glycerol-3-phosphate dehydrogenase, a kind of terminal differentiation marker, is essential for glycerol-3-phosphate synthesis to induce the formation of TG (Lien et al., 2007). The 


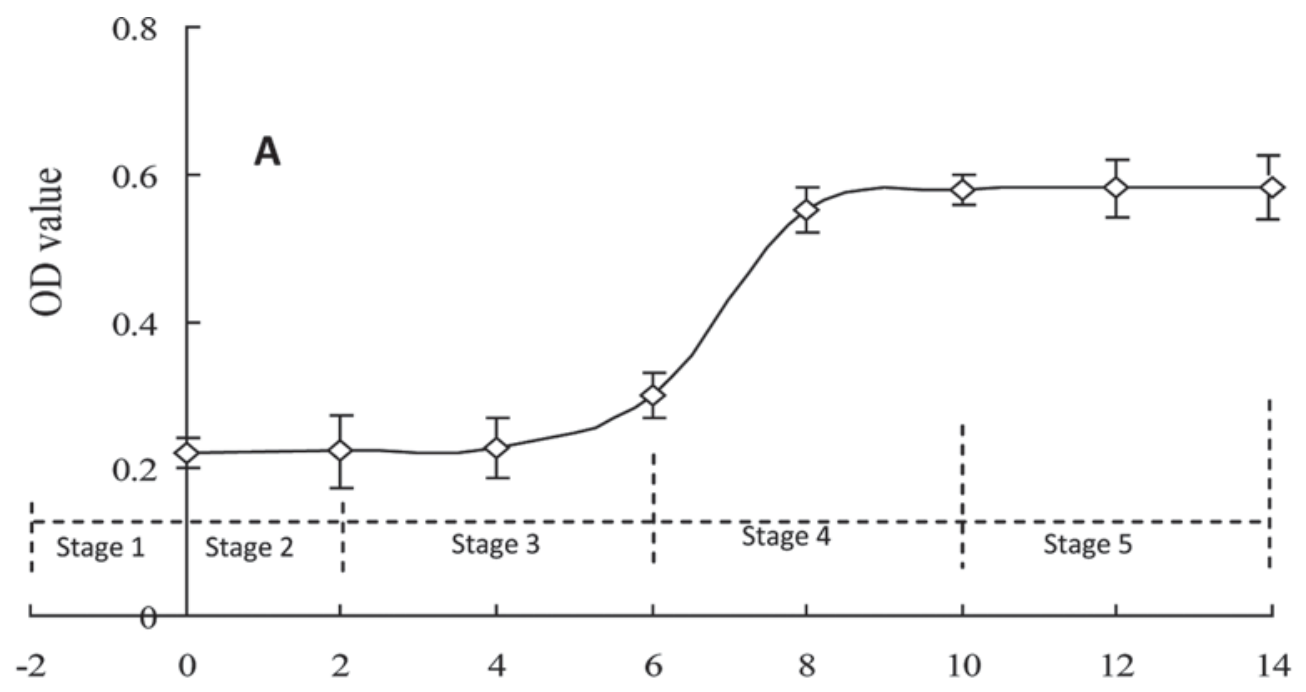

Days after differentiation

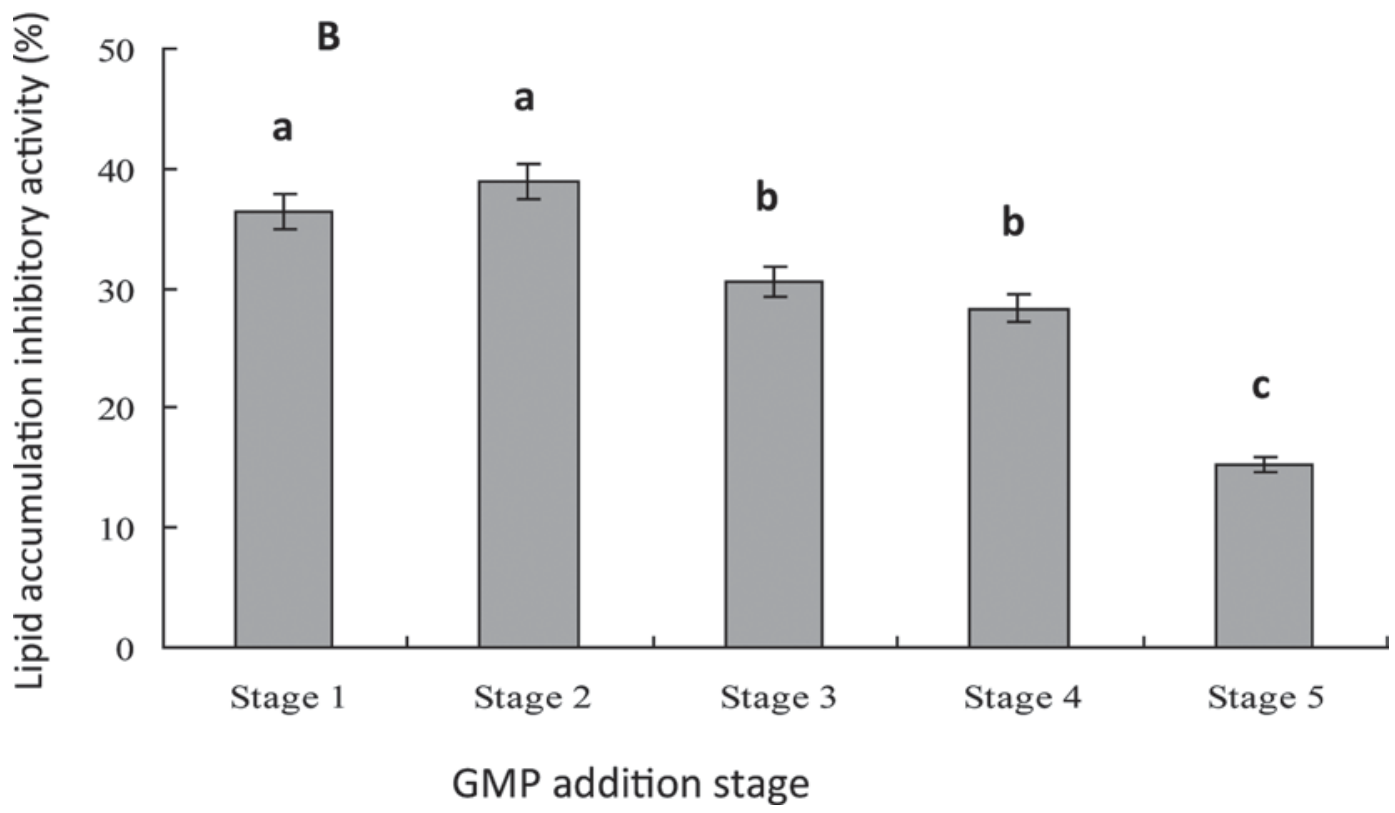

Figure 4. The natural differentiation curve of adipocytes without casein glycomacropeptide (GMP) supplementation (A; OD = optical density). Casein glycomacropeptide $(2.5 \mathrm{mg} / \mathrm{mL}$ ) was added to cell cultures at different stages: stage 1 (from $2 \mathrm{~d}$ before differentiation induction: i.e., d -2 to d 0), stage 2 (from d 0 to 2), stage 3 (from d 2 to 6 ), stage 4 (from d 6 to 10), and stage 5 (from d 10 to 14). The effect on lipid accumulation was quantified (B). Results are presented as means \pm SD of 3 replicated cultures. Columns that do not share the same letter are significantly different from each other at $P<0.05$.

reduction of GPDH activity was also observed in the cultures treated with GMP in the present study. All of these may indirectly demonstrate that GMP possesses FAS-inhibiting activity.

It is now accepted that not only the lipid content, but also the number of adipocytes, is variable in adult mammals. Apoptosis plays an important role in the regulation of adipose mass. Adipocyte number may be increased by differentiation of preadipocytes, a process which has been widely studied (Cornelius et al., 1994; Prins and O'Rahilly, 1997). The decrease of lipid accumulation was more evident for treatments that consisted of incubating the 3T3-L1 cells with xanthohumol, a Humulus lupulus L. prenylflavonoid, from confluence (d -2 until d 2 of differentiation; Mendes et al., 2008). Cultures treated with activin A, a member of the transforming growth factor- $\beta$ superfamily, during the late phase was less effective than the treatment 

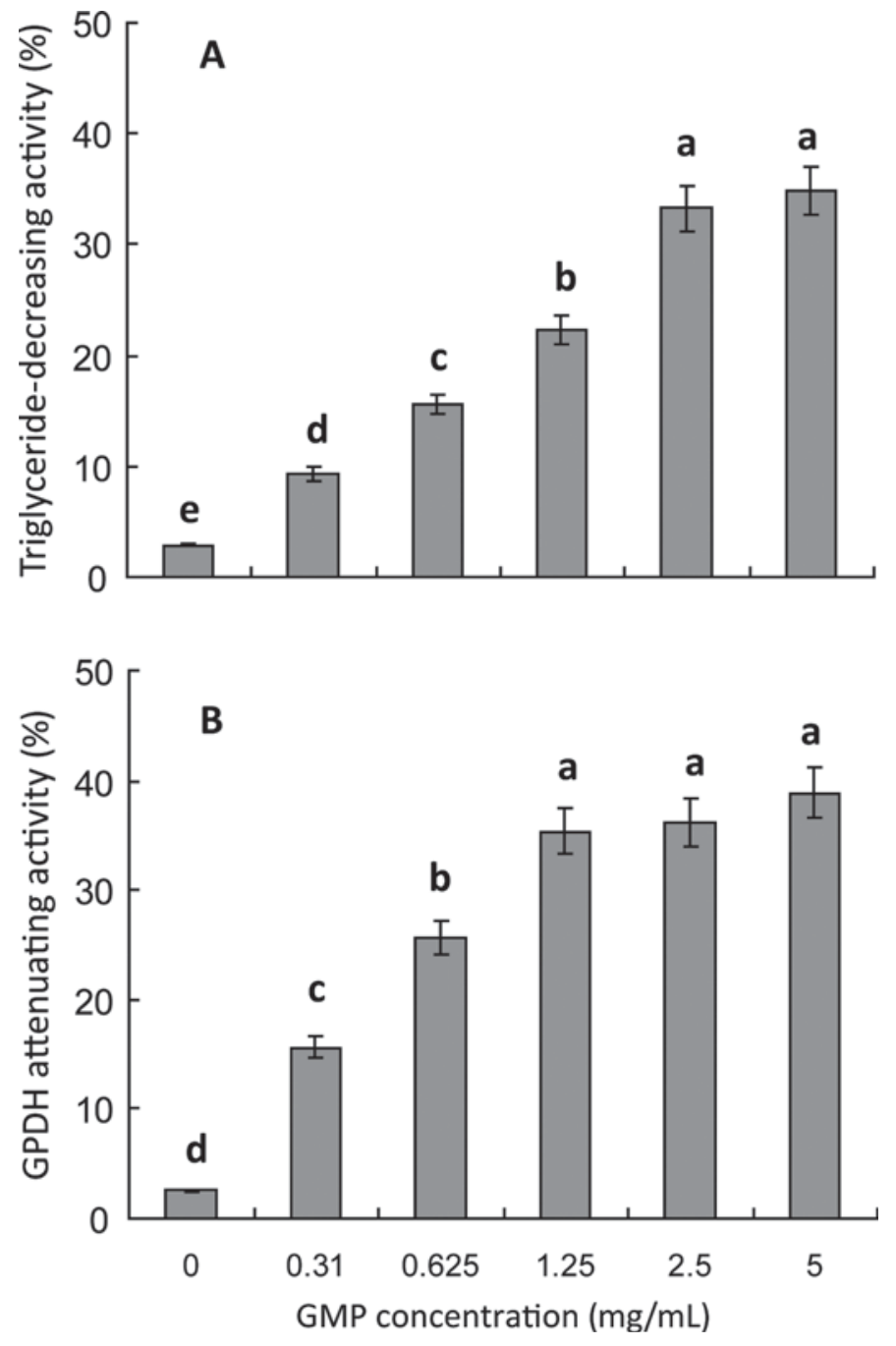

Figure 5. Effect of casein glycomacropeptide (GMP) supplementation at concentrations from 0 to $5.0 \mathrm{mg} / \mathrm{mL}$ on glycerol-3-phosphate dehydrogenase (GPDH) activity and triglyceride production of preadipocytes. Results are expressed as means \pm SD of triplicate experiments. Columns that do not share the same letter are significantly different from each other at $P<0.05$.

during the early phases (Hirai et al., 2005). The present study shows that the inhibition activity of GMP to the proliferation and differentiation of rat preadipocytes was dependent on the phase of GMP addition. Adding GMP at the lag phase of preadipocyte proliferation, as well as before or at the beginning of differentiation, was more efficient than addition at other phases. This means that GMP addition time is very important and the antidifferentiation effect of GMP occurs in the early differentiation stages.

\section{CONCLUSIONS}

The proliferation, differentiation, and lipid accumulation of rat preadipocytes in vitro is significantly inhib- ited after being treated with GMP. The effect was more pronounced with a treatment time of $72 \mathrm{~h}$ than were other co-incubation periods. In addition, the decrease of lipid accumulation was the most effective for treatment of preadipocytes with GMP from $2 \mathrm{~d}$ before differentiation induction to within $2 \mathrm{~d}$ after differentiation began. Casein glycomacropeptide not only decreased the proliferation and differentiation of preadipocytes, but also attenuated lipid accumulation and the GPDH activity of adipocytes. These results demonstrate that GMP supplementation efficiently suppresses adipogenesis in rat preadipocytes and adipocytes. Therefore, further research can focus on the potential antiobesity effect of GMP on obese rats and human subjects induced by a high-fat diet.

\section{ACKNOWLEDGMENTS}

This study was financially supported by the National Natural Science Foundation of China (Grant No. 30972291) and the National High Technology Research and Development Program (863 Project, 2008AA10Z320) of the Ministry of Science and Technology of China. We thank Zhongquan Dai and Yinghui Li (Beijing astronaut training center) for technical assistance; Scott Gualco (California Polytechnic State University) for his critical reading of the manuscript.

\section{REFERENCES}

Anderson, G. H., and S. E. Moore. 2004. Dietary proteins in the regulation of food intake and body weight in humans. J. Nutr. 134:974S-979S

Aziz, A., and G. H. Anderson. 2007. The effect of dairy components on food intake and satiety: Mechanisms of actions and implications for the development of functional foods. Pages 19-45 in Functional Dairy Products. Vol. 2. M. Saarela, ed. Woodhead Publishing Ltd., Cambridge, UK.

Azuma, N., K. Yamauchi, and T. Mitsuoka. 1984. Bifidus growthpromoting activity of a glycomacropeptide derived from human к-casein. Agric. Biol. Chem. 48:2159-2162.

Belobrajdic, D. P., G. H. McIntosh, and J. A. Owens. 2004. A high whey-protein diet reduces body weight gain and alters insulin sensitivity relative to red meat in Wistar rats. J. Nutr. 134:14541458.

Brück, W. M., S. L. Kelleher, G. R. Gibson, G. Graverholt, and B. L. Lönnerdal. 2006. The effects of alpha-lactalbumin and glycomacropeptide on the association of $\mathrm{CaCo}-2$ cells by enteropathogenic Escherichia coli, Salmonella typhimurium and Shigella flexneri. FEMS Microbiol. Lett. 259:158-162.

Burton-Freeman, B. M. 2008. Glycomacropeptide (GMP) is not critical to whey-induced satiety, but may have a unique role in energy intake regulation through cholecystokinin (CCK). Physiol. Behav. 93:379-387.

Cornelius, P., O. A. MacDougald, and M. D. Lane. 1994. Regulation of adipocyte development. Annu. Rev. Nutr. 14:99-129.

Forest, C., P. Grimaldi, D. Czerucka, R. Negrel, and G. Ailhaud. 1983 Establishment of a preadipocyte cell line from the epididymal fat pad of the lean C57 BL/6J mouse - Long term effects of insulin and triiodothyronine on adipose conversion. In Vitro 19:344-354.

Furuyashiki, T., H. Nagayasu, Y. Aoki, H. Bessho, T. Hashimoto, K. Kanazawa, and H. Ashida. 2004. Tea catechin suppresses adipocyte differentiation accompanied by down-regulation of PPAR- 
gamma2 and C/EBPalpha in 3T3-L1 cells. Biosci. Biotechnol. Biochem. 68:2353-2359.

Garaulet, M., J. J. Hernandez-Morante, J. Lujan, F. J. Tebar, and S. Zamora. 2006. Relationship between fat cell size and number and fatty acid composition in adipose tissue from different fat depots in overweight/obese humans. Int. J. Obes. (Lond.) 30:899-905.

Guilloteau, P., I. Le Huërou-Luron, J. A. Chayvialle, R. Toullec, M. Legeas, C. Bernard, L. Roger, and F. Mendy. 1994. Effect of caseinomacropeptide (CMP) on gastric secretion and plasma gut regulatory peptides in preruminant calves. Reprod. Nutr. Dev. 34:612-613.

Guo, W., J. K. Choi, J. L. Kirkland, B. E. Corkey, and J. A. Hamilton. 2000. Esterification of free fatty acids in adipocytes: A comparison between octanoate and oleate. Biochem. J. 349:463-471.

Hall, W. L., D. J. Millward, S. J. Long, and L. M. Morgan. 2003. Casein and whey exert different effects on plasma amino acid profiles, gastrointestinal hormone secretion and appetite. Br. J. Nutr. 89:239-248.

Hirai, S., M. Yamanaka, H. Kawachi, T. Matsui, and H. Yano. 2005. Activin A inhibits differentiation of 3T3-L1 preadipocyte. Mol. Cell. Endocrinol. 232:21-26.

Hsu, C. L., S. L. Huang, and G. C. Yen. 2006. Inhibitory effect of phenolic acids on the proliferation of 3T3-L1 preadipocytes in relation to their antioxidant activity. J. Agric. Food Chem. 54:41914197.

Hsu, C. L., and G. C. Yen. 2007. Effects of flavonoids and phenolic acids on the inhibition of adipogenesis in 3T3-L1 adipocytes. J. Agric. Food Chem. 55:8404-8410.

Keogh, J. B., B. W. Woonton, C. M. Taylor, F. Janakievski, K. Desilva, and P. M. Clifton. 2010. Effect of glycomacropeptide fractions on cholecystokinin and food intake. Br. J. Nutr. 104:286-290.

Kim, Y.-J., Y.-K. Oh, S.-S. Yoo, K.-Y. Park, W. Kang, and S. Park. 2005. Antiobesity effect of recombinant human caseinomacropeptide in Sprague-Dawley rat. Biotechnol. Bioprocess. Eng. 10:242247

Kuhajda, F. P., L. E. Landree, and G. V. Ronnett. 2005. The connections between $\mathrm{C} 75$ and obesity drug-target pathways. Trends Pharmacol. Sci. 26:541-544.

Lam, S. M., P. J. Moughan, A. Awati, and H. R. Morton. 2009. The influence of whey protein and glycomacropeptide on satiety in adult humans. Physiol. Behav. 96:162-168.

Li, J. J., C. J. Huang, and D. Xie. 2008. Anti-obesity effects of conjugated linoleic acid, docosahexaenoic acid, and eicosapentaenoic acid. Mol. Nutr. Food Res. 52:631-645.

Lien, T.-F., C.-P. Wu, and Y.-M. Horng. 2007. Chromium picolinate depressed proliferation and differentiation of 3T3-L1 preadipocyte. Nutr. Res. 27:176-180.

Lowry, O. H., N. J. Rosebrough, A. L. Farr, and R. J. Randall. 1951. Protein measurement with the Folin phenol reagent. J. Biol. Chem. 193:265-275.

Ma, L., K. S. Ge, F. Z. Ren, and X. Y. Mao. 2008. Study on optimization of ultrafiltration separation conditions of casein glycomacropeptides from whey protein concentrate. Food Sci. (Chinese) 29:278-282.

Martinez-Villaluenga, C., V. P. Dia, M. Berhow, N. A. Bringe, and E. Gonzalez de Mejia. 2009. Protein hydrolysates from beta-congly- cinin enriched soybean genotypes inhibit lipid accumulation and inflammation in vitro. Mol. Nutr. Food Res. 53:1007-1018.

Mendes, V., R. Monteiro, D. Pestana, D. Teixeira, C. Calhau, and I. Azevedo. 2008. Xanthohumol influences preadipocyte differentiation: Implication of antiproliferative and apoptotic effects. J. Agric. Food Chem. 56:11631-11637.

Miguel, M., M. A. Manso, R. López-Fandiño, M. J. Alonso, and M. Salaices. 2007. Vascular effects and antihypertensive properties of א-casein macropeptide. Int. Dairy J. 17:1473-1477.

Morikawa, K., M. Nonaka, H. Mochizuki, K. Handa, H. Hanada, and K. Hirota. 2008. Naringenin and hesperetin induce growth arrest, apoptosis, and cytoplasmic fat deposit in human preadipocytes. J. Agric. Food Chem. 56:11030-11037.

Mosmann, T. 1983. Rapid colorimetric assay for cellular growth and survival: Application to proliferation and cytotoxicity assays. J. Immunol. Methods 65:55-63.

Pichon, L., M. Potier, D. Tome, T. Mikogami, B. Laplaize, C. MartinRouas, and G. Fromentin. 2008. High-protein diets containing different milk protein fractions differently influence energy intake and adiposity in the rat. Br. J. Nutr. 99:739-748.

Pilvi, T. K., R. Korpela, M. Huttunen, H. Vapaatalo, and E. M. Mervaala. 2007. High-calcium diet with whey protein attenuates body-weight gain in high-fat-fed $\mathrm{C} 57 \mathrm{Bl} / 6 \mathrm{~J}$ mice. Br. J. Nutr. 98:900-907.

Prins, J. B., and S. O'Rahilly. 1997. Regulation of adipose cell number in man. Clin. Sci. 92:3-11.

Rahman, A., S. G. Kumar, S. W. Kim, H. J. Hwang, Y. M. Baek, S. H. Lee, H. S. Hwang, Y. H. Shon, K. S. Nam, and J. W. Yun. 2008. Proteomic analysis for inhibitory effect of chitosan oligosaccharides on 3T3-L1 adipocyte differentiation. Proteomics 8:569-581.

Rajic, A., J. Dhulia, C. G. Hosking, and D. J. Autelitano. 2010. A novel dairy-derived isolate that inhibits adipogenesis and significantly reduces weight gain in a high fat animal model. Int. Dairy J. 20:480-486.

Royle, P. J., G. H. McIntosh, and P. M. Clifton. 2008. Whey protein isolate and glycomacropeptide decreases weight gain and alters body composition in male Wistar rats. Br. J. Nutr. 100:88-93.

Torii, I., S. Morikawa, A. Nakano, and K. Morikawa. 2003. Establishment of a human preadipose cell line, HPB-AML-I: Refractory to PPARgamma-mediated adipogenic stimulation. J. Cell. Physiol. 197:42-52.

Wang, Y. W., and P. J. Jones. 2004. Conjugated linoleic acid and obesity control: Efficacy and mechanisms. Int. J. Obes. Relat. Metab. Disord. 28:941-955.

Wise, L. S., and H. Green. 1979. Participation of one isozyme of cytosolic glycerophosphate dehydrogenase in the adipose conversion of 3T3 cells. J. Biol. Chem. 254:273-275.

Wu, Z., N. L. Bucher, and S. R. Farmer. 1996. Induction of peroxisome proliferator-activated receptor gamma during the conversion of 3T3 fibroblasts into adipocytes is mediated by $\mathrm{C} / \mathrm{EBPbeta}, \mathrm{C} /$ EBPdelta, and glucocorticoids. Mol. Cell. Biol. 16:4128-4136.

Yvon, M., S. Beucher, P. Guilloteau, I. Le Huerou-Luron, and T. Corring. 1994. Effects of caseinomacropeptide (CMP) on digestion regulation. Reprod. Nutr. Dev. 34:527-537. 